\title{
The biology of human sexuality: evolution, ecology and physiology
}

P W Bateman \& N C Bennett

(University of Pretoria)

\section{ABSTRACT}

The biology of human sexuality: evolution, ecology and physiology

Many evolutionary biologists argue that human sexual behaviour can be studied in exactly the same way as that of other species. Many sociologists argue that social influences effectively obscure, and are more important than, a reductionist biological approach to human sexual behaviour. Here,we authors attempt to provide a broad introduction to human sexual behaviour from a biological standpoint and to indicate where the ambiguous areas are. We outline the evolutionary selective pressures that are likely to have influenced human behaviour and mate choice in the past and in the present; ecological features that influence such things as degree of parental care and polygamy; and the associated physiology of human sexuality. Then they end with a discussion of 'abnormal' sexuality.

\section{SEXUAL SELECTION}

Males and females of many species of animal are very different in appearance. Male birds are often more colourful than their female counterparts, have longer tails and produce distinctive sex-specific calls or songs. Male mammals in turn may possess elaborate tusks, antlers, horns, manes or other obvious indicators of their gender. Charles Darwin attempted to explain these striking sexual dimorphisms, dicromatisms, and behaviour patterns in his book "The Descent of Man, and Selection in Relation to Sex" (1871) with what he called 'sexual selection'. 'Natural selection', the mechanism by which the engine of evolution produces new species, and the subject of Darwin's famous book "The Origin of the Species by Means of Natural Selection” (1859), preserves and selects for features that allow individuals to survive. Sexual selection attempts to explain the presence of apparently survival costly features such as long tails and bright colours. Here the selective pressure is the female who chooses to mate with only the most attractive and ornamented males. In Darwin's words, females are 'coy' and males are 'ardent'. 


\section{ANISOGAMY}

These differences in male and female behaviour are considered to stem from a simple thing: anisogamy. Anisogamy refers to the difference in size of the sex cells, or gametes. Males of many taxa produce multiple, motile, tiny sperm cells while females produce only a few, large, immobile, nutrient-loaded egg cells. In fact, we can define 'males' as those members of a species that produce microgamete seekers (sperm), and females as those that produce macrogamete providers (eggs). So how does behaviour correlate to size of sex cells?

\section{LIFETIME REPRODUCTIVE SUCCESS}

A J Bateman (1948) showed that the lifetime reproductive success (RS) of male fruit flies increased with the number of females with which they mated. In other words, the more females a male mates with, the more eggs he fertilises, and as a consequence, the more offspring he produces. For the female, with her limited supply of eggs, the sperm from one or two males may be sufficient to fertilise her entire potential reproductive output (lifetime RS). For her, therefore, multiple mating is unnecessary. This dichotomy is referred to as Bateman's Principle: “there is a stronger correlation, in males (relative to females) between number of mates and fertility (number of progeny)".

\section{SPERM COMPETITION}

'Hogamous, higamous; /Men are polygamous; /Higamous, hogamous; Women monogamous', goes the ditty of psychologist William James. Nevertheless, it is not that simple. Females of many species do actively seek out multiple partners with which to mate. How can this be explained? If females have limited lifetime reproductive success potential in comparison to that of males, we can predict that they will be choosier (Darwin's 'coy') than males. One method to ensure that their precious eggs are fertilised by the best available males is to promote competition between the males. This can happen before matings, for example two stags jousting, or after mating, when the ejaculates of two or more males compete internally for the fertilisation of the ova. This is called 'sperm competition' and it is believed that this is a powerful selective force on male and female morphology and behaviour. For instance, a male who can produce a much larger ejaculate than his rivals may be able to out-compete those rivals by a simple lottery. There will be selection, therefore, to 
evolve large testes to produce large numbers of sperm, and large epididymes (the storage tubules behind the testes) to store the ejaculate. A large penis would be adaptive in that it could place the ejaculate as close to the site of fertilisation as possible. In turn, there will be selection on females to evolve a longer genital tract, or a more hostile internal environment (e.g. $\mathrm{pH}$ of the vaginal secretions) to exclude inferior sperm, or the ability to eject ejaculates of certain males.

\section{Homo sapiens}

Does any of this apply to humans? Knowing what we do about anisogamy, sexual selection, parental investment and sperm competition in other species can we make any predictions about the sexual behaviour of Homo sapiens through a comparative study?

Firstly, humans are sexually dimorphic (size and body shape differences between men and women), more than some great apes (by which we mean other members of the Family Hominidae: the gorilla, the common chimpanzee, the bonobo or pygmy chimpanzee, and the orang-utan), and less than in others. Men are generally taller and heavier than women; they have more upper body strength, higher metabolic rates, more facial and body hair, deeper voices, larger brains, higher infant mortality, later sexual maturity and die younger. In comparison to the other members of the Hominidae, humans have less hairy bodies but proportionally hairier heads (men also have beards and male-pattern baldness, but this can be seen in at least one geographical race of common chimpanzee as well), whiter eyes, longer noses, larger ear lobes, more everted lips, smaller less dangerous teeth, more expressive faces and more dexterous hands. Human males have bigger penises than other great apes and human females demonstrate continuous sexual receptivity and apparent concealed ovulation. These features, it is claimed by some, are sexually-selected, or at least, are exapted to be used as sexual signals (an adaptation is a functional feature that is produced by Darwinian selection; an exaptation is the same except that it now has a function for which it was not originally selected for. E.g., feathers may have evolved as a thermoregulatory adaptation, but have now been exapted for flight). Support for this comes from the observation that most of these features develop, or are exaggerated when humans reach puberty, are displayed during sex, are apparently valued as attractive features in many cultures and are enhanced selectively by clothes and makeup. It must be emphasised that this is an 
evolutionary psychology argument. Evolutionary psychology (EP) is adaptationism applied to the human mind and attempts to explain "...aspects of human behaviour, and thence culture and society, on the basis of universal features of human nature that found their final evolutionary form... 100-600,000 years ago" (Rose \& Rose 2001). $\mathrm{EP}$ is a compelling and exciting theory, but it does not receive unalloyed support, with many researchers (from the social and natural sciences) rejecting even its most basic premises (see Rose \& Rose 2001 for examples).

So, when we examine the sexual behaviour of human beings, where are the borders between 'pure' biology and the shadowy zone of cultural epiphenomena? We know that human beings are anisogamous: the human egg is 85,000 times larger than the human sperm cell. We know that men produce large ejaculates (an average of $2.75 \mathrm{ml}$, containing 280 million sperm: about two trillion in a reproductive life of 60 years) and females produce one egg per lunar month (13 per year: about 500 in a reproductive life of 40 years). We know that record lifetime reproductive success is enormously different between men and women. The beloved example of evolutionary biologists is the Emperor Moulay Ismail the Bloodthirsty of Morocco (born c. 1645, reigned from 1672 to 1727) who is credited with producing 888 children through a kind of battery-farm, harem system (virgins in at menarche, out on a full pension at 30 years old). The total is debated, but still very high. The record for a woman (from the Guinness Book of Records) is the less well-remembered "wife of Feodor Vassilyev" who, in the $18^{\text {th }}$ Century, produced 69 children from 27 pregnancies. Both examples are extremes, but serve to illustrate the Bateman Principle.

\section{ADAPTATIONISM}

Beyond this, we cannot be so confident. The degree of dimorphism and the size of the testes indicate that, biologically, Homo sapiens is a mildly polygynous (single males mating with multiple females) ape with moderate levels of sperm competition. In other words, certain men will have higher reproductive success than others will by monopolising women and women will quite often 'cuckold' their social partner by mating with other men. Such a reductionist statement would infuriate most social scientists, and in fact, it is too reductionist for most evolutionary biologists as well. In this chapter, however, we will consider the evidence from an evolutionary, 
adaptive standpoint, but clearly indicate where it is felt that the evidence is equivocal.

\section{Development of sexuality in male and female Homo sapiens}

Humans become sexually mature when they enter puberty and become fertile. For both males and females, this is due to hormonal events that are triggered by physiological condition. For girls it is generally considered that they need to exceed about $50 \mathrm{~kg}$ in weight before they can enter menarche. Importantly, young women need to exceed a certain percentage of body fat before menstruation can be maintained. A sixteen-year-old woman must have over about $20 \%$ body fat to be fertile. Interestingly, there is some evidence that many pre-technical societies are fully aware of this, and girls about to enter puberty are encouraged to eat more to hasten the event and to ensure high fertility.

In current mainstream societies, especially Western ones with increasing levels of obesity, the age of the onset of menarche has been declining, with many girls menstruating from between ten and twelve years old. In the $17^{\text {th }}$ century, it was about seventeen years old in Europe. Leptin, a hormone produced by fat, kick-starts the process that will wake up the ovarian follicles. Early maturation has raised some worries: it has been described as "starting a car engine without a skilled driver". In Kalahari San people, with limited calorie intake, and low fat reserves, menarche onset can be at up to nineteen years of age.

At puberty, the hypothalamus starts to produce gonadotrophinreleasing hormone $(\mathrm{GnRH})$ that stimulates the pituitary to secrete hormones that, in turn, initiate the production of oestrogen and testosterone in the gonads. At the same time, the gonads begin the conveyor belt of sperm and egg production. Much is often made of the fact that, from birth, a baby girl has a stock of ovarian follicles already present in her tiny gonads and the description of this is often associated with negative terminology such as how this 'stockpile' 'degenerates' up to menopause at about 50 years old. In fact, although spermatogenesis continues throughout a male's reproductive life, the sperm-producing tissue has also been present from before birth, waiting for the hormonal kiss of life.

Hormone production not only influences fertility, it also affects the outward appearance of boys and girls. Both sexes have androgens and oestrogens, but in different ratios. At about thirteen or 
fourteen when boys experience a testosterone peak from their testes making them look more like adult men, they are actually experiencing their second dose of testosterone. The first occurs in the womb, at about two months into development as the testes differentiate from the foetal proto-gonads. This testosterone-burst ensures that at birth the parents are able to identify their child as a son (we shall return to this). The second burst of testosterone produced by their gonads at about fourteen results in all the classic symptoms of puberty: growth spurts, body hair, body odour, and the development of an 'adult' face. Testosterone encourages the growth of the lower face and brow ridges, both indicators of 'masculinity'. A higher ratio of oestrogen in girls suppresses this growth but apparently induces growth of the lips, a 'feminine' indicator. Girls born with a male twin are the only ones that are exposed to testosterone in the womb, and according to some studies, are more 'masculine' in appearance and behaviour, but what exactly constitutes 'male behaviour' can be strongly culturally influenced. It certainly influences female twins in other species: in cattle, such females are known as 'freemartins' - masculinised females.

The onset of puberty, ovulation and sperm production does not mean that young humans are truly 'adult', and most of our cultural mores reflect this. Unlike most animals (though we should add the caveat that we do not know enough about other great apes, and other socially complex animals like elephants to be entirely exclusive here) pubertal humans have yet to reach their full fertility. A thirteen year old boy may be a raging mess of hormones and sperm production, and a twelve year old girl may be gaining an 'adult' female shape and menstruating, but peak fertility is not reached until about nineteen years old (slightly later for men than for women).

\section{Mate choice in Homo sapiens}

So now we have young adult Homo sapiens with all the hallmarks of masculinity and femininity written upon their faces and bodies because of the hormones that have made them fertile. Is there any evidence that these indicators have a role in sexual selection? In women, studies have shown that a small lower jaw and the lower part of the face are regarded as attractive by men. In men, studies have shown that a large lower jaw regarded as dominant by women. As these are indicators of 'femininity' and 'masculinity', this is highly suggestive of a role in sexual selection. Even more interesting is that these high dominance indicators in highly masculine men are 
not perceived by women to be as attractive as lower dominance men. According to questionnaires, high dominance men are more violent, initiate more fights and initiate more sexual encounters (based on a sample that is Western and predominantly Caucasian). If we take a purely behavioural ecology standpoint here, this makes sense as we know that females of many taxa avoid high dominance males, due to costs to their own fitness in associating with them (more testosterone means more bullying). In humans, as usual, we must be careful due to cultural influences.

What other features might influence mate choice? Humans like symmetry. Faces that are more symmetrical are more attractive to us. Babies like faces and particular components that signal human faces: curves, strong contrasts of light and dark, moving lips. Babies will stare longer at more symmetrical, more 'attractive' faces than at 'unattractive' faces. Maybe, as most people's faces are relatively symmetrical then symmetrical faces look more human, and hence we like them. However, it has been suggested in multiple studies that symmetry also reflects 'developmental stability'. In other words, the more symmetrical the features (and this can apply to any bilaterally symmetrical structure: hands, feet, legs, arms, fingers etc.) the 'fitter', the 'healthier', the individual would be. In a world where sexual selection acts upon individuals, we could predict that symmetry could have a role in mate choice. In humans, symmetry appears to predict male sexual behaviour. Men who are more symmetrical report attracting more sexual partners, have sexual intercourse earlier in life, have more partners outside of their primary relationship, tend to have more offspring, tend to have fewer serious diseases, are heavier for their height and are more muscular.

Can symmetry potentially act as a cue in choosing mates, or at least influence our choice in mates? A study has found that women preferred symmetrical male faces in black and white photographs, even though they could not judge differences in symmetry themselves. They even preferred symmetrical faces when only one side of the face was presented.

Other studies have tested whether body odour can be used as a test of symmetry. In a study that has become a discussion favourite in all evolutionary ecology journal club meetings subject males slept in a t-shirt for three consecutive days, which were then given to women to evaluate the smell. Fascinatingly there was a positive 
relationship for attractiveness of smell and higher symmetry. This relationship was only found when the women evaluating this were at their most fertile period of the menstrual cycle.

Can men differentiate between women based on odour? Another study tested whether men can judge by body odour if women are fertile (which would be adaptive). In the same sort of t-shirt test it was found that men preferred the scent of women who were in their follicular phase of the menstrual cycle and therefore ovulatory and fertile.

Other features that have been posited as indicators of high 'quality' in women include the oft-mentioned Waist to Hip ratio (WHR) which, it has been suggested, acts as a cue of fertility, as low WHR (which translates crudely as a curvaceous body) is supposed to correspond with optimal fat distribution for high fertility. Also apparently important is the Body Mass Index (BMI): weight scaled to height. In its broadest sense then, a good choice is a curvaceous woman neither too thin nor too fat to compromise fertility. Once again, despite the generality of this conclusion, we find suggestive support in that most female models fall within a very narrow range of BMI and WHR and this range has stayed constant in models over several decades.

So far, so fascinating after-dinner anecdote. Once again, we must insert the caveat that not everyone is totally convinced by studies involving odorous t-shirts, based as they are on samples from Western University campuses.

If two humans have selected each other for mates, whether based on facial symmetry, body odour, hip to waist ratio or muscularity, or a shared dislike of chocolate ice cream, what happens then?

\section{Orgasm, intromission and conception}

One area of human sexuality that always arouses interest is the orgasm. For men it is relatively easy to 'explain': orgasm is always associated with ejaculation. That it is pleasurable can be seen as secondary, but of course, it is no great intuitive leap to see how such pleasure is a possible exaptation. The female orgasm, with no ejaculation, is more difficult to explain. Female orgasm is not limited to Homo sapiens; certainly, chimpanzees experience it. The most parsimonious explanation for female orgasm is that, just as the clitoris is a homologue of the penile glans, so is the female orgasm a 
homologue of the necessary male orgasm: in other words it is merely an epiphenomenon, a side effect of parallel development of males and females. In support of this view, it is pointed out how rarely the female orgasm occurs from intercourse (a third of women in a British study reported never, or rarely, having an orgasm during intercourse). Another view is that, regardless of the male and female orgasm originally being 'mere' homologues, there can still be selection for satisfying, pleasurable female orgasms if they help to ensure a strong pair bond between sexual partners. In the pygmy chimpanzee, or bonobo (Pan paniscus), females will rub their clitorises together to climax. The bonobo clitoris is much larger than that of humans, and orgasms in this species do seem to act as social bonding device.

Physiologically, it has been suggested that the orgasm may have a very clear adaptive function in that it stimulates the mouth of the cervix to dip into an ejaculate deposited in the vagina, aiding uptake and hence likelihood of fertilisation. Against this argument is that for this to happen the female orgasm has to occur after the male orgasm (unless the cervical movements continue after the orgasm) and most women (Western, Caucasian) report that their orgasms usually occur before that of their partner.

Depressingly, the primatologist Sarah Blaffer Hrdy (1999) has pointed out that, even, if orgasms do function to "dispel tension and strengthen bonds between partners", for the vast majority of women in much of the world, living in aggressively patriarchal societies, orgasms, reflecting libido and sexual assertiveness are more likely to get a woman punished, beaten or killed. Many societies have customs such as veiling, purdah, and, worst of all, genital mutilation through clitoridectomy (total or partial excision of the clitoris) and infibulation (sewing together of the vaginal lips). If there is any kind of selection in action here, Hrdy suggests that the female orgasm may once have been an adaptation, but now is no longer selected for, and will disappear.

To fertilise the egg, whether 'up-sucked' by an orgasm or not, the sperm has to be first deposited in an ejaculate in the vagina. There seems to have been different selection pressures in different species; just as the human clitoris is much smaller than that of the bonobo, so the human penis is much larger than those of the other great apes. It has been suggested that this may be a consequence of 
sperm competition. In a series of experiments with false vaginas and penises where the shape and size of the glans and coronal ridge (the head of the penis) had been altered, it was shown that the human penis could displace semen already present in the vagina. In turn, the long penis of Homo sapiens could be an adaptation to deposit the ejaculate deep in the vagina where it would be less susceptible to displacement.

For sperm competition to occur in humans, a woman would need to have intercourse with two men within three to six days of each other. Although sperm can live this long in the genital tract, many spermatozoa in the ejaculate will have died, and the man who has more sperm present at optimal time of conception is likely to become the father. With the discovery that blood types can be used to assign paternity came the realisation that many children tested are not the offspring of the husband. To be crudely evolutionary minded, this makes complete sense: why shouldn't a woman attempt to ensure higher reproductive success for herself by mothering multiple children of different paternity and foisting them on a highprovisioning cuckold? In the 1980s, discovery of hyper-variable DNA meant that 'genetic finger-printing' allowed researchers to assign paternity almost unambiguously. Immediately it was realised that most monogamous species were in fact only socially monogamous and that polyandry and 'extra-pair copulations' were extremely common. Once again, we must emphasise that what makes a woman 'cuckold' her husband may be (and probably is) very different to what makes a female thrush engage in extra-pair copulations.

To fertilise an egg, sperm has to present when a woman is fertile. In a great ape, like the common chimpanzee (Pan troglodytes), the period of greatest fertility is clearly marked by pale crimson swellings of the anal and perineal skin. The primatologist Jane Goodall memorably dubbed these female chimps 'pink ladies', and, together with their behaviour (solicitation of sex, following males), peak fertility is obvious to the male chimps. Women, however, do not exhibit a periodic oestrus with a clear analogue of the chimps' swelling. Instead, they have a bloodstained vaginal discharge known as the mensus. It is argued whether, if men can tell by odour when a woman is fertile (as discussed above), human females have true concealed ovulation. At any rate, it is not obvious as in other great apes. 
Menstruation in women is typically, but not always, once a lunar month. Menstruation normally occurs for between 4-5 days and this marks the end of the life of the corpus luteum (a structure in the ovary used to maintain the vascularisation and thickened lining of the uterine wall and assist in the maintenance and support of a conception). The first day of menstruation can be considered day 1 of the new oestrous cycle. The development of a new follicle(s) in the ovary follows the onset of menstruation and is associated with the development of a primordial follicle into a mature Graafian follicle; this lasts about 2 weeks but can be extremely variable. Developing follicles are associated with rising levels of oestrogen that in turn feed back to higher centres in the brain (the hypothalamus) to promote further release of a hormone from the pituitary called follicle stimulating hormone (FSH) that in turn brings about the maturation of the follicle.

Interestingly, at a particular concentration the oestrogen that feeds back to the hypothalamus becomes inhibitory and promotes the release of luteinizing hormone $(\mathrm{LH})$ from the pituitary that results in ovulation or the release of an egg from the Graafian follicle.

After ovulation has occurred, a corpus luteum begins to form and secretes large amounts of progesterone. A corpus luteum may last for between 8 and 14 days. If fertilization occurs then the corpus luteum is retained.

In the event that fertilisation does not take place the corpus luteum starts to regress and breakdown, the uterine lining or endometrium is suddenly deprived of progesterone and the endometrial arteries go into spasm and the endometrial lining is sloughed off together with blood. This endometrial sloughing seems an extreme response to failed fertilisation, and it has been suggested that it might be an adaptation to the potential pathological effects of sperm and copulation, which might have introduced viral and bacterial infective agents.

\section{Homo sapiens babies and birth}

Nine months of gestation and human babies are born. Unlike every other great ape, the human baby has a head bigger than the mother's pelvic outlet. A long labour for a gorilla is about twenty minutes. Human mothers are considered to have had an 'easy' labour at eight hours. Associated with this, mortality of both mothers and babies at birth can be high. With selection for large brains (perhaps due to a 
high protein diet in ancestral Homo species), and a limitation on pelvic outlet size due to our bipedalism, human babies are less well developed than other great apes at birth - 'exterogestate foetuses'. This means that humans have to care for their offspring for much longer than other great apes.

An immediately obvious difference between human newborns and other great apes is how fat human babies are. Newborn chimps appear painfully skinny and vulnerable to humans used to fatswaddled neonates. A human baby is about $16 \%$ fat by weight compared to about $2 \%$ for monkeys. Why this is, when all simian milk is about the same at a low $4 \%$ fat (discussed below), is not fully understood. The primatologist Sarah Blaffer Hrdy (1999) outlines four main theories:

1. Fat acts as insulation. Human babies are born naked and grow no warming layer of fur.

2. Fat is an insurance policy. If a baby has to be left for an extended period, then fat will help it survive. Hrdy cites the story of a neonate that survived for a week on its fat in an earthquake-ruined building. If this were true, however, then why do other great apes not do the same?

3. Fat feeds the brain. With a brain of 1,400 сc (450 in apes), the fat is a stockpile for rapid development of the expensive to produce brain tissue. Fifty \% of the total basal metabolic rate of a baby goes to brain development. Born with large brains already, babies will increase their brain to $70 \%$ of its adult size within a year of birth, and the swaddling of neonatal fat helps to feed this growth.

4. Finally, there is what Hrdy calls 'Advertisement for myself'. Fattening up before birth, rather than after, actually endangers both mother and infant: it makes passage through the birth canal even tighter and more perilous. This is well known enough for women in many cultures to starve in pregnancy to ensure a smaller baby. Hrdy points out that this is the mother's way of countering a process otherwise controlled by the foetus. The foetus directs the nutrient flow rate from mother to placenta to itself, and this allows the baby approaching birth to fatten up immensely. A baby born fat (and fat equals viable) is advertising itself to its mother as a healthy, brainy offspring with a high chance of survival. In all likelihood, the mother 
already has a semi-dependent previous offspring with whom the newborn has to compete, and so the baby has a selective pressure to be 'adorable' (a fat, bouncing baby). This is a blatantly adaptive story, but a compelling one none the less.

\section{Ecology of Homo sapiens and child survival}

Infant Homo sapiens, adorable or not, are born into a world in which they desperately need assistance to thrive. They rely primarily on their mother, but also on others, to survive. It is difficult to raise children to adulthood. An estimated 36\% of parturient! Kung San women die with no surviving offspring. What provisioning does the mother provide?

Like all mammals, women produce milk from mammary glands to feed their baby. Human milk is quite dilute in comparison to that from other species (88\% water and $4 \%$ fat), which reflects the fact that human babies are rarely far from the nipple and suckle on an ad hoc basis. Mammals that hide their offspring and return at periods to suckle them briefly have richer milk, as do species with a short period of lactation (that of seals is more like $60 \%$ fat). This constant supply of milk, albeit dilute, is obviously a huge cost to women ('a tooth for every child', according to the old saying). This is why the fat the mother put down at puberty, which triggered the production of leptin, entry into menarche and the start of fertility is so important. This vital fat has been laid down in female-specific areas: the buttocks, upper thighs and abdomen. In addition, of course, fat is laid down in the breasts, though larger breasts do not mean more milk, and the fat here is usually not metabolised to produce milk. Why human females develop large breasts early, and retain them even when not lactating, is not fully understood, though, once again, they could have been exapted to advertise youth and fertility.

Only mothers provide milk: is help from the father needed? Human offspring are dependent for a long time, and there may be two offspring simultaneously reliant: something that rarely occurs in other great apes. One would imagine that input (direct provisioning or otherwise) from the father would be highly important, but most studies show that the role of the father varies greatly within and between cultures and the prevailing ecology will influence decisions made by both parents on whether to provision or desert their offspring. 
The anthropologist Monique Borgerhoff Mulder (1991) identified three important socio-ecological factors that influence how much care a parent will provide:

1. How safe is the environment? The dangerous environment of a South American rainforest (snakes, parasites, rain) means that foragers of the Ache culture provide very intensive levels of parental supervision. Interestingly, this high degree of care (carrying the infant etc.) results in very slow development of gross motor abilities in Ache children.

2. What is the level of nutritional stress? Children in poor nutritional state are more likely to die. Once weaned, biparental care will be favoured in environments where survival is dependent on parental care. In some cultures, with sex-based division of labour fathers (or other male relatives) simply have to be present to provide calories, e.g.! Kung San people in marginal environments.

3. Are alternative caretakers either available or suitable? Caretaking (allo-mothering) by relatives is common in some human groups. Nevertheless, its acceptance varies across cultures. Kenyan Kipsigis people rely heavily on sibling caretakers to relieve that mother. Micronesian Ifalukese women who have first-born daughters use them as caretakers for later offspring. South American foraging Ache people, however, do not consider caretaking as safe for the dependent child. Relatives can assist in other ways. South American crop-raising Ye'kwana women who are nursing receive assistance in crop growing by female kin. In addition, the degree of gender-based division of labour can have an effect on who the caretakers or allo-mothers are. In most societies, caretakers tend to be female. In the Aka pygmies of central Africa, hunting is primarily by netting and is done equally efficiently by men or women. Men, therefore, spend as much time in direct offspring care (holding, cleaning, playing with, and teaching) as the women. The men will even allow babies to suck on their nipples as a natural pacifier. Amongst San people, men hunt away from home, and for long periods: their direct contact with children is much less than that of the women.

If an Ache child's father dies before it reaches fifteen, its chance of mortality increases. Fatherless Ache children sometime die by 
infanticide, as other group members do not want to provision it. Again, there are distinct differences culturally, depending on the ecology of the group. Infanticide may occur in foraging Ache but never in agro-pastoral Kipsigis where provisioning comes from the extended family.

\section{Ecology of Homo sapiens and mating systems}

If we were to be resolutely evolutionary in our approach, we would use the term 'mating systems'. More familiarly, when speaking of humans, we would say 'marriage patterns'. In Western cultures, we assume that (socially and legally at least) monogamous marriages are the norm. If we look across multiple traditional cultures, we find that, in fact, monogamy occurs in only about $16 \%$ of them. Polygyny (a single 'husband' with multiple 'wives') makes up the bulk, with about $83 \%$, and polyandry (a single 'wife' with multiple 'husbands') makes up the remaining tiny balance. So, what are the ecological pressures that result in these differences in mating system?

According to the anthropologist Laura Betzig (1992), “across space and time, polygyny has overlapped with despotism, monogamy with egalitarianism". In other words, human polygyny is what behavioural ecologists would call resource-defence polygyny. Men compete for power or resources that will give them access to women. To be able to do this, the society in which they live must be ordered in such a way as to allow them to obtain and maintain more resources than other men. As societies became stratified (labourers, merchants, aristocracy) an unequal distribution of resources inevitably occurred, and with it immense variation in male reproductive success. At the peak are men like Moulay Abdul of Morocco, who, with access to nearly all the resources of north west Africa, was able to create a harem of hundreds of women. It is worth making the distinction though, that to Moulay Abdul and other absolute rulers, the women themselves are a resource to be hoarded. Originally, we could posit, polygyny arose because certain men were better able to provide for women and children than others were. !Kung San are considered to be monogamous, but some men, better hunters than others, can be opportunistic polygynists, exchanging resources (e.g. protein) directly for sex. That polygyny is not common, or permanent, in such hunter-gatherers is due to the marginal environment in which they live (and the consequent codification of social mores). Sometimes there are simply not enough resources to allow polygyny to occur. Then a pair-bonded 
monogamous relationship would be more stable. Agro-pastoral Kipsigis men, buffered by agriculture, are able to maintain a polygynous culture: there is a direct correlation between how many acres of land each farmer owns and how many reproductive wives per married year.

Why then, with effectively unlimited resources, is monogamy the norm in mainstream Western cultures? First, as should be clear by now, social monogamy (marriage) is not the same as sexual monogamy. Secondly, semi-divorced from their environment as they are, Western cultural marriage patterns are more likely to be influenced by historical inertia, religious mores and socio-political factors. As mentioned above, monogamy also tends to occur in marginal environments that support small groups, but even then is plastic in response to resource availability.

Polyandry, the rarest form of marriage pattern, is usually fraternal. Brothers, rather than split inherited land into ever-smaller packages, combine the land and their families. The most familiar example is that of Tibetan peasants in Ladakh. Here, the important environmental variable is the cultivable land available: small areas of usable land that would become unviable if split into small units. In practice, although a woman may socially be married to three brothers, it is usually only the eldest who fathers children; the younger ones become priests or attempt to practice adultery with other men's wives. In addition, it is plastic. As soon as a man is able to accumulate resources in some other way (e.g. through trade), then he will become a polygynist.

\section{Is there such a thing as abnormal sexuality?}

The famous sex biologist Kinsey claimed that up to $10 \%$ of the male population of the U.S.A. were mainly or exclusively homosexual. This is now considered as a high estimate and that exclusive homosexuals make up about one in fifty of a population, and this is comparable between populations.

Homosexuality can be perceived as adaptive if one considers kin selection. The point being, one does not only have to produce offspring (descendent kin) to ensure genetic survival; one could also assist in raising the offspring of one's relatives (non-descendent kin, also bearing one's genes).

However, what is 'homosexuality' and, even if it is found in all populations, is it 'abnormal'? Certainly, it is common in many 
species; over three hundred vertebrates apparently display same-sex courtship and genital contact (Bagemihl 1999). However, there is also a semantic problem here: if homosexuality does occur in $2 \%$ of any given human population then, statistically, it is not normal. Statistically 'normal' then implies normality. In other words 'not normal', means 'abnormal', means 'not right'. We must guard against this implication when considering emotive subjects like homosexuality. Is it, therefore, viable to consider such behaviour 'unnatural'?

Joan Roughgarden (2004), an evolutionary biologist, has produced a compelling thesis in which she claims that the argument for 'unnaturalness' of same sex sexuality can be dispelled by dropping Sexual Selection (Darwin 1871) for her own 'Social Selection'. As simply as possible, what Social Selection says is that sex is not just about exchanging gametes, but about forming bonds within societies and negotiating for access to resources necessary to reproduce. The argument then follows that this negotiation will take place as much within sexes as between sexes. If sex is primarily about relationships and 'power games' influencing lifetime RS and secondarily about gamete exchange then that is why much more sex than reproduction occurs. For instance, a couple with two children, who have enjoyed a fifty-year monogamous relationship with an average of twice-weekly intercourse, have had 2,700 copulations per child. That is why non-reproductive sexual behaviour such as homosexuality occurs, because, according to social selection, homosexuality ultimately functions in the same way as heterosexuality: negotiating social bonds and access to resources.

Regardless of the above, what evidence is there of a genetic component to homosexuality? One study found that if one dizygotic male twin is homosexual, his brother has a one in four likelihood of also being homosexual. If one monozygotic male twin is homosexual, his identical brother has a $50 \%$ of also being homosexual. If true, this alone suggests that there is a sizeable genetic component to homosexuality. There is also some evidence that homosexuality is inherited from the maternal (X chromosome), not paternal line (i.e. homosexual uncles are more likely to be from the maternal side of the family).

The pursuit of a 'gay gene' is sociologically problematic. Traditionally, those on the right of the political spectrum have 
embraced genetic determinism while those on the left have repudiated it. With homosexuality, the positions have generally been reversed. Those on the left have accepted the 'gay gene' as support that homosexuals should not be persecuted for their lifestyle, while those on the right have rejected it, claiming that homosexuals 'choose' to follow their lifestyle, or that it is environmentally (socially) induced. The geneticist Steve Jones (2002), however, quotes the "vile headline" of the right wing British newspaper The Daily Mail: “Abortion Hope after Gay Gene Finding”.

Other aspects of 'abnormal' sexuality come from one's personal observation that most people are clearly either male or female. Some people may be more 'feminine', some more 'masculine'. This is intuitive, and most of us are aware that men are genetically different from women (XY chromosomes versus XX). In fact, the two sexes are closer than many of us might care to think. As foetuses developing in the womb, both males and females develop along very similar lines until a certain point. This is reflected in our physiology: both sexes have nipples; in males, they are not, generally, functional (although useful to Aka fathers). Both sexes have the same basic genital plumbing: clitoris and glans, ovaries and testes. The genital structure of women, with a vaginal opening bordered by labia majora and minora is mirrored in the male penis. Until puberty, and for many men, beyond, there is a visible dark line along the base of the penis, known as the median raphe. This is the 'sealed' homologue of the female genital slit.

This differentiation begins in the womb when male foetuses begin to produce testosterone and, for a while, develop faster than female foetuses. Remember that at puberty the testes again produce a burst of testosterone. For some children, genetically male, the testes do not produce a burst of testosterone in the womb and the foetus continues to develop along the 'female' plan. Born with undescended testes, unfused scrotal tissue like labia and reduced penises, these boys appear indistinguishable from girls and are often raised as such. With puberty, the testes produce testosterone, facial hair grows, the penis often enlarges, and the testes attempt to descend. Semen is also produced, ejaculating from beneath the penis. In the Dominican Republic, this condition is familiar enough for the boys to be known as 'guevedoche', or 'penis-at-twelve' (in fact the average age of 'becoming male' is at about sixteen). It was found that, of eighteen guevedoche on which data existed, thirteen 
became socially male at puberty, and went on to marry women, two died, one was asexual and avoided people, one felt herself to be biologically and socially female and married a man and one was ambiguous about his/her status.

Once again, such an 'abnormal' sexuality is abnormal in the sense that it is statistically extremely uncommon, but where gender identification is a sliding scale (multiple types of intersexual) rather than a bi-categorical state (male: female), it is not to be unexpected. Analogously, the literature is full of observations on other species where sexual role switching takes place, even gender identity. Many group-living fish compete to become males; if the 'male' dies, the dominant 'female' changes colour, switches from egg to sperm production and fertilises the other females. However, is that comparable to what we see in human societies?

Roughgarden (2004) gathers an impressive review of apparent abnormal intersexual behaviour across cultures. She emphasises the difference between traditional cultures that view 'gender- and sexuality-variant' people positively or neutrally and the modern Western view that, although often accepting in its outlook, has a history of defining physical and behavioural sexual ambiguity as pathological. She gives examples such as 'Two-spirited' people in North American Indian groups (both men and women who flout traditional roles, e.g. warrior women who take female lovers), 'Mahu' in Polynesia (boys who are raised amongst and as women) and 'Hijra' in the Indian sub-continent (a religious caste/sect made up of men-to-women transgenders).

An in-depth discussion of such groups is beyond the remit of this article, but it is worth emphasising again, what has been evident throughout that it is difficult to have any certainties about human sexuality, whether as biologists or sociologists. Science, of course, revels in uncertainty: it is one of its greatest attributes. Judgement, condemnation and conservatism have no place here.

\section{Consulted literature}

Bagemihl, B 1999. Biological Exuberance. Animal Homosexuality and Natural Diversity. London: Profile Books.

Bateman, A J 1948. Intra-sexual selection in Drosophila. Heredity 2, 349-368.

Betzig, L 1992. Roman polygyny. Ethology and Sociobiology 13, 309-49.

Blaffer Hrdy, S 1999. Mother Nature. Natural Selection and the Female of the Species. London: Chatto \& Windus. 
Borgerhoff Mulder, M 1991. Behavioural ecology of humans: Studies of foraging and reproduction, in: Krebs J R and Davies N B (eds.), Behavioural Ecology, 3rd Edition. Blackwell Scientific Publications, 69-98.

Cartwright J 2000. Evolution and Human Behaviour. Darwinian Perspectives on Human Nature. Basingstoke: Palgrave.

Darwin C 1859. The Origin of the Species by Means of Natural Selection. London: John Murray.

-, 1871. The Descent of Man, and Selection in Relation to Sex. London: John Murray.

Grammer K, Fink B, Møller A P, \& Thornhill R 2003. Darwinian aesthetics: sexual election and the biology of beauty. Biological Review 78, 385-407.

Jones S 2002. Y: The Descent of Men. London: Abacus.

Kappeler P, Van Schaik C (eds) 2004. Sexual Selection in Primates. New and Comparative Perspectives. Cambridge: Cambridge University Press.

McGraw K J 2002. Environmental predictors of geographic variation in human mating preferences. Ethology 108, 303-317.

Ridley M 1993. The Red Queen. Sex and the Evolution of Human Nature. London: Penguin.

Roughgarden J 2004. Evolution's Rainbow. Diversity, Gender, and Sexuality in Nature and People. Berkeley: University of California Press.

Rose H \& Rose S (eds) 2001. Alas poor Darwin. Arguments Against Evolutionary psychology. London: Vintage, Random House.

Thornhill R, \& Gangestad SW 1996. The Evolution of Human Sexuality. Trends in Ecology and Evolution 11, 98-102. 